\title{
Inactivation of the fliY gene encoding a flagellar motor switch protein attenuates mobility and virulence of Leptospira interrogans
} strain Lai

\author{
Sumei Liao, ${ }^{1,2}$, Aihua Sun ${ }^{3}$, David M Ojcius ${ }^{4}$, Senlin $\mathrm{Wu}^{3}$, Jinfang Zhao ${ }^{2}$ and
} Jie Yan*2

Address: ${ }^{1}$ College of Life Sciences, Zhejiang University, Hangzhou 310058, PR China, ${ }^{2}$ Department of Medical Microbiology and Parasitology, College of Medicine, Zhejiang University, Hangzhou 310058, PR China, ${ }^{3}$ Zhejiang Medical College, Hangzhou 310053, PR China and ${ }^{4}$ Health Sciences Research Institute and School of Natural Sciences, University of California, Merced, CA 95343, USA

Email: Sumei Liao - lsmplum@yahoo.com.cn; Aihua Sun - sunah@zjmc.net.cn; David M Ojcius - dojcius@ucmerced.edu; SenlinWu - keyanchu1999@126.com; Jinfang Zhao - 3021851002@zju.edu.cn; Jie Yan* - med_bp@zju.edu.cn

* Corresponding author

Published: 9 December 2009

BMC Microbiology 2009, 9:253 doi:10.1 186/147|-2180-9-253
Received: 29 March 2009

Accepted: 9 December 2009

This article is available from: http://www.biomedcentral.com/I47I-2180/9/253

(c) 2009 Liao et al; licensee BioMed Central Ltd.

This is an Open Access article distributed under the terms of the Creative Commons Attribution License (http://creativecommons.org/licenses/by/2.0), which permits unrestricted use, distribution, and reproduction in any medium, provided the original work is properly cited.

\begin{abstract}
Background: Pathogenic Leptospira species cause leptospirosis, a zoonotic disease of global importance. The spirochete displays active rotative mobility which may contribute to invasion and diffusion of the pathogen in hosts. FliY is a flagellar motor switch protein that controls flagellar motor direction in other microbes, but its role in Leptospira, and paricularly in pathogenicity remains unknown.
\end{abstract}

Results: A suicide plasmid for the fliY gene of Leptospira interrogans serogroup Icterohaemorrhagiae serovar Lai strain Lai that was disrupted by inserting the ampicillin resistance gene (bla) was constructed, and the inactivation of fliY gene in a mutant (fliY) was confirmed by PCR and Western Blot analysis. The inactivation resulted in the mRNA absence of fliP and fliQ genes which are located downstream of the fliY gene in the same operon. The mutant displayed visibly weakened rotative motion in liquid medium and its migration on semisolid medium was also markedly attenuated compared to the wild-type strain. Compared to the wild-type strain, the mutant showed much lower levels of adhesion to murine macrophages and apoptosis-inducing ability, and its lethality to guinea pigs was also significantly decreased.

Conclusion: Inactivation of fliY, by the method used in this paper, clearly had polar effects on downstream genes. The phentotypes observed, including lower pathogenicity, could be a consequence of fliY inactivation, but also a consequence of the polar effects.

\section{Background}

The genus Leptospira is composed of both saprophytic and pathogenic species [1]. Pathogenic Leptospira spp., such as L. interrogans, L. borgpetersenii, L. weilii and L. kirschner, are the causative agents of leptospirosis, a serious world-wide disease in humans and animals [2,3]. The disease in humans occurs mostly after contact, often through skin wounds, with soil or water contaminated by urine of infected animals. Its severity varies from mild to rapidly fatal. Severe symptoms are characterized by visible jaun- 
dice involving hepatic injury, acute renal failure, carditis and hemorrhage, and case fatality varies from a few percent to $25 \%$ [3-6]. However, the mechanisms of disease caused by pathogenic Leptospira spp. remain largely unknown.

Both pathogenic and saprophytic leptospires express two endoflagella (periplasmic flagella). One of the endoflagella is attached at one end of the cell and is located between the protoplasmic cylinder and the outer membrane sheath [7-9]. The endoflagella, rotating within the periplasmic space, are responsible for spirochete motility. In pathogenic Leptospira species, this motility is considered to contribute to invasion into hosts and diffusion within the hosts during infection $[9,10]$. In previous studies, we found that pathogenic leptospires can adhere to host cells with one or two termini of the microbial bodies, while non-pathogenic leptospiral strains lacked this ability $[11,12]$. The adhering positions were located at the terminal knobs in which flagellar basal bodies are found $[1,7]$. At the bottom of the flagellar structure, there is a basal body composed of MS and C rings $[13,14]$. In flagellated bacteria, some proteins in the Fli family form the $\mathrm{C}$ ring, which functions as the flagellar rotor and contains the directional switching capability of the flagellar motor [15-18]. However, a possible role for the leptospiral endoflagella in pathogenicity has never been explored.

A complete set of flagella-associated genes were found in the genomic sequences of $L$. interrogans serovar Lai strain Lai and serovar Copenhageni strain Fiocruz L1-130, including four genes that encode flagellar motor switch proteins (FliG, FliM, FliN and FliY) $[19,20]$. In bacteria, the flagellar motor switch proteins play a critical role in control of flagellar motor direction $[14,17,18]$. Thus far FliY has been found in some spirochetes and a few bacteria but does not exist in most bacteria $[21,22]$. Particularly, FliY of Bacillus subtilis was shown to be a CheY-Phydrolyzing protein in the chemotactic signaling cascade [22]. In addition, leptospiral FliY carries a carboxy-terminal domain of 60 amino acid residues that is homologous to a domain of YscQ in Yersinia pestis $[19,20]$. The YscQ protein was identified as a member of the flagellar associated type III secretion system (T3SS), with multiple functions such as controlling the directional rotation of flagella and the export of virulence factors including Yop proteins $[23,24]$. The $\mathrm{C}$ ring of Escherichia coli does not have FliY, but its FliN has a high sequence homology with FliY of L. interrogans strain Lai [19] and FliN is an essential agent for motility and virulence protein export [25]. These data suggest that FliY of pathogenic Leptospira species may have important functions in motility and virulence.

In the present study, we constructed a fliY gene knock-out (fliY-) mutant of L. interrogans serovar Lai strain Lai based on homologous recombination using a suicide plasmid. To examine the possible role of FliY in pathogenesis, the mutant and wild-type strain were compared in assays of motility in liquid medium and migration on semisolid agar, adhesion to macrophages, stimulation of apoptosis in infected host cells, and lethality to guinea pigs.

\section{Results \\ Products of fliY gene amplification and rFliY expression}

The amplification segments with expected size of the entire fliY gene (1065 bp) from L. interrogans serovar Lai strain Lai were obtained by PCR (Fig 1A). The cloned fliY gene had $100 \%$ nucleotide sequence identity with the reported sequences in GenBank (Accession No.: NC 004343, NC 005823) [10,11]. The recombinant plasmid, E. coli BL21DE3PET32a-fliY, expressed rFliY under inducement of isopropyl- $\beta$-D-thiogalactopyranoside (IPTG), and the purified rFliY by Ni-NTA affinity chromatography showed a single band on a polyacrylamide gel after electrophoresis (Fig 1B). The rabbits immunized with rFliY could produce rFliY-specific serum antibody and the immunodiffusion titer of antiserum against rFliY was $1: 4$.

\section{Characterization of the fliY- mutant}

To create a fliY- mutant of $L$. interrogans, we cloned the $f l i Y$ gene into p2NIL and inserted an ampicillin gene at the $B g l$ II site near the 5 ' end. This plasmid was then introduced into $L$. interrogans followed by selection for ampicillin resistance, to create a fliY bla mutant. Sequencing data indicated that the fliY gene and ampicillin resistance gene (bla) segments in suicide plasmid p2NIL flir-bla had the same orientation, and the nucleotide sequences were the same as in the original cloned fliY and bla genes. The fliYmutant could grow in $100 \mu \mathrm{g} / \mathrm{ml}$ ampicillin-contained Korthof liquid medium for at least 3 months in our labo-


\section{Figure I}

Amplification and expression of the fliY gene and purification of the rFliY protein. Panel $A$, showing PCR analysis. Lane I: DNA marker (TaKaRa, China); lane 2: the amplification segment of the entire fliY gene; lane 3: blank control. Panel B, showing SDS-PAGE analysis. Lane I: protein marker (TaKaRa); lane 2: pET32a with no insertion of the fliY gene; lane 3: the expressed recombinant protein, rFliY; lane 4: the purified rFliY protein. 
ratory. The generation time of the mutant (about $10 \mathrm{~d}$ ) was the same as that of the wild-type strain. Subsequent PCR analysis confirmed that the mutant maintained a modified fliY gene that was larger (2019 bp) than the wild-type gene (1065 bp), into which inserted the ampicillin resistance gene (954 bp) had been inserted (Fig 2A). The Western Blot analysis also revealed the absence of expression of FliY in the mutant (Fig 2B). Furthermore, the absence of mRNAs for the fliP and fliQ genes, downstream of fliY gene, indicated that the transcription of the two genes were inhibited (data not shown). In fact, ten genes (fliY, LA2612, fliP, fliQ, fliR, flhB2, flhA, flhF, LA2605 and LA2604) should be transcribed by the same operon, based on the genome structure predicted by the software, MicrobesOnline Operon Predictions (Fig 3).

\section{Persistently lower motility of the fliY- mutant}

Normally, leptospires have a typical motive manner with rotation. However, all microbes of the fliY- mutant in liquid Korthof medium by dark-field microscopy only had $40 \%$ of rotative motion frequency per minute of the wildtype strain, but presented a similar shape to the wild-type strain (data not shown). On semisolid Korthof agar plates, the colonies of the fli $Y^{-}$mutant were noticeably

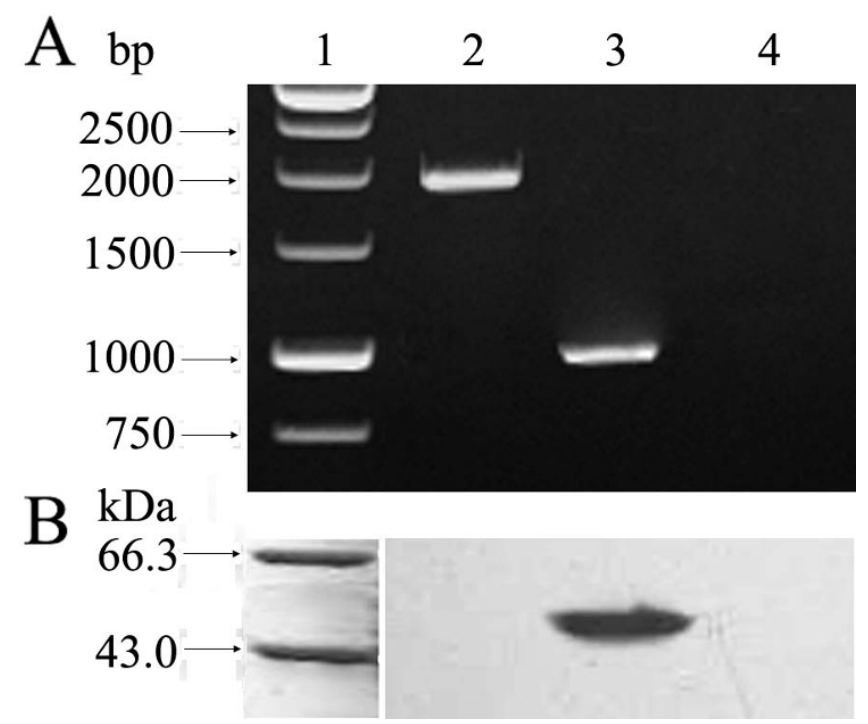

\section{Figure 2}

Confirmation for insertion mutantion of fliY gene in the fliY- mutant. Panel A, showing PCR analysis. Lane I: DNA marker (TaKaRa); lane 2: the amplification segment (2019 bp) of mutated fliY gene from the fliY- mutant; lane 3: the amplification segment (I065 bp) of the fliY gene from the wild-type strain; lane 4: blank control for PCR. Panel B, showing Western Blot analysis. Lane I: protein marker (TaKaRa); lane 2: the fliY- mutant lacking the FliY protein; lane 3: the wild-type strain expressing the FliY protein; lane 4: blank control for Western Blot assay. rFliY antiserum was used as the primary antibody. smaller (2-3 $\mathrm{mm}$ in diameter) than that of the wild-type strain (6-8 $\mathrm{mm}$ in diameter) (Fig 4), consistent with attenuated motility of the mutant.

\section{Altered adhesion of the fliY- mutant}

The wild-type L. interrogans strain Lai could adhere to the surface of J774A.1 cells with one or both bacterial ends (Fig 5A). The attached wild-type leptospires were visible on the cell surface after 10 min post inoculation (p.i.) and the adhesion ratios approached a plateau after 40 to 60 min p.i. (Fig 6). However, the fli $Y^{-}$mutant was significantly impaired in its ability to adhere to the macrophages, compared to the wild-type strain $(P<0.05)$ (Fig 5B and Fig. 6).

\section{Host-cell apoptosis induced by the wild-type and the fliY- mutant strains}

As shown in Fig 6, the wild-type L. interrogans strain Lai induced apoptosis of J774A.1 cells, and the maximal apoptotic ratio $(48.2 \pm 2.9 \%)$ appeared after $4 \mathrm{~h}$ coincubation, as detected by flow cytometry (Fig 7A). However, the ability of the $f l i \mathrm{Y}^{-}$mutant to cause apoptosis was markedly decreased, and the levels of apoptosis and late apoptosis/necrosis at all the different incubation times were significantly lower than those induced by the wild-type strain $(P<0.05)$ (Fig $7 \mathrm{~B}$ and $7 \mathrm{C})$.

\section{Attenuated lethality of the fliY-mutant strain in guinea pigs}

The lethality to guinea pigs of the wild-type L. interrogans strain Lai was significantly larger than of the fli $Y^{-}$mutant during a 10d post-challenge period (Table 1). No animals infected by the fli $Y^{-}$mutant strain died comparing with $100 \%$ death, which were infected by wild-type strain with the same dosage. When the challenge dosage for the fli $Y^{-}$ mutant was increased to ten times the dosage used for the wild-type strain, only $60 \%$ of the animals infected with the fliY- mutant died.

\section{Discussion}

Recent reports have shown that flagellin and other flagella-associated proteins from many bacteria participate in adhesion to host cells and colonization of hosts [26-28]. In vitro studies have suggested that the role of flagella could be to increase invasion into host cells and survival within macrophages [29,30]. However, the correlation between flagella and pathogenicity of pathogenic Leptospira spp. had not been investigated until now. L. interrogans serogroup Icterohaemorrhagiae serovar Lai strain Lai is the most prevalent pathogenic leptospiral strain, which is responsible for over $70 \%$ of human leptospirosis cases in China [31]. We therefore inactivated the fliY gene in $L$. interrogans strain Lai using a suicide plasmid, which is a frequently adopted strategy for determining the function of a target gene. Recently, Croda and his colleagues used 


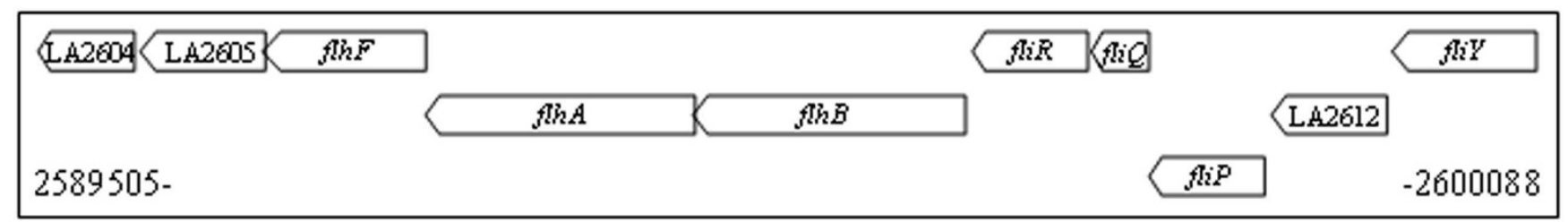

\section{Figure 3}

Genes present with the fliY gene within the same predicted operon. Annotation of the genes (gene name/product): fliY/flagellar motor switch protein; LA26 I 2/flagellar protein required for flagellar formation; fliP/flagellar biosynthesis protein; fliQ and fliR/flagellar biosynthetic proteins and type III secretion apparatus proteins; flhB/flagellar protein; flhA/flagellar biosynthesis protein; flhF/flagellar GTP-binding protein; LA2605/ParA protein; and LA2604/hypothetical protein.

plasmid pB2SK to successfully construct a suicide plasmid with spectinomycin resistance for inactivating the ligB gene of $L$. interrogans serovar Copenhageni strain Fiocruz L1-130 [32]. In the present study we first used another plasmid, p2NIL, with an ampicillin resistance gene (bla) to construct a fliY gene knock out $\left(f l i Y^{-}\right)$mutant. A fli $Y^{-}$ mutant has been constructed, but that fliY inactivation by ampicillin cassette insertion also negatively affected downstream genes; therefore, care has to be taken when interpreting the phenotypes observed for this mutant.

The inactivation of the fliY gene has shown different effects on formation of flagella in different bacteria. In Bacillus subtilis, the deletion of fliY resulted in the loss of flagella [33]. However, the flagella were still produced in the fliY-deleted strain of Bacillus cereus [34]. Although the leptospiral $\mathrm{fli}^{-}$- mutant generated in this study displayed remarkably attenuated motility compared to the wild-type strain, it maintained the typical spiral shape and propeller movement which is caused by the periplasmic endoflagella $[1,7]$. As mentioned previously, the major function of flagellar motor switch proteins is to control flagellar
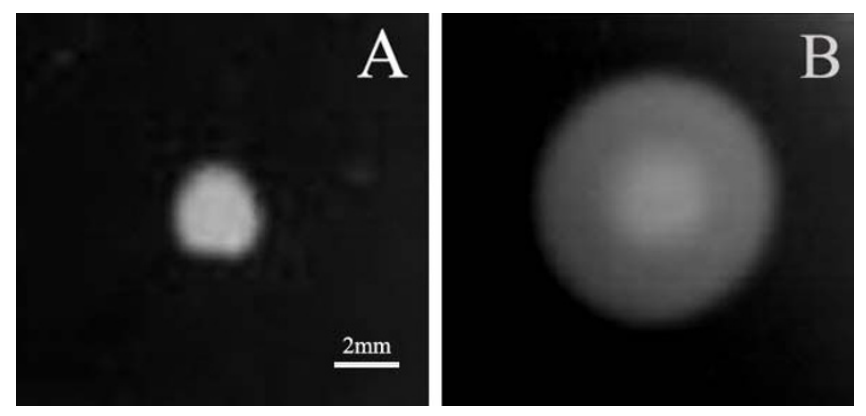

Figure 4

Colony sizes of the fliY-mutant and wild-type strain on semisolid Korthof agar. The colonies with different sizes formed by the fliY-mutant (A) and wild-type strain (B) on semisolid Korthof agar. The leptospires were cultured on $8 \%$ RS semisolid Korthof plate for three weeks. This experiment was repeated three times. motor direction $[16,19-22]$. Thus, we infer that the fliY gene inactivation should not affect the formation of the endoflagella.

It is well known that adhesion to host cells is a primary and critical step for bacterial infection [35,36]. Recently, the importance of cell adhesion for pathogenic Leptospira spp. has been demonstrated $[11,12,37,38]$. Adhesion to host cells also acts as an essential role for pathogenicity of other spirochetes $[39,40]$. Mononuclear macrophages are the most important phagocytes in the human innate and acquired immnune systems. However, many pathogenic bacteria can evade host immunity by inducing apoptosis of macrophages [41-43]. Similarly, pathogenic Leptospira spp. can escape from the host immune system by promoting macrophage apoptosis $[11,44-46]$. In the present study, we provide evidence that the ability of the fliYmutant to adhere to J774A. 1 cells, to induce apoptosis in the cells, and to cause death in guinea pigs is much lower than for the wild-type strain. All the phentotypes observed, including lower pathogenicity, could be a consequence of fliY inactivation, or a consequence of the polar effects, or of both.

T3SS is one of protein export systems used by most Gramnegative bacteria [47]. Morphologically, as a transmembrane channel, T3SS is composed of multiple protein complexes called an injectisome, responsible for transporting virulence factors into host cells, some of which cause cell metabolic disorder and death [47-49]. However, the flagellar export apparatus can also function as a bacterial virulence protein secretion system [50]. For example, FliF of Pseudomonas aeruginosa, a flagellar associated protein component in the MS ring, is involved in adhesion by controlling secretion of bacterial adhesins [51]. Although the T3SS and flagellar export apparatus are two relatively separate systems in many pathogenic bacteria [52], the T3SS and flagellar export apparatus in Yersinia enterocolitica play a common role in secretion of bacterial phospholipases during infection [53]. Taken together, these observations suggest that inactivation of the leptospiral fliY gene (or of the downstream located fliPQ 


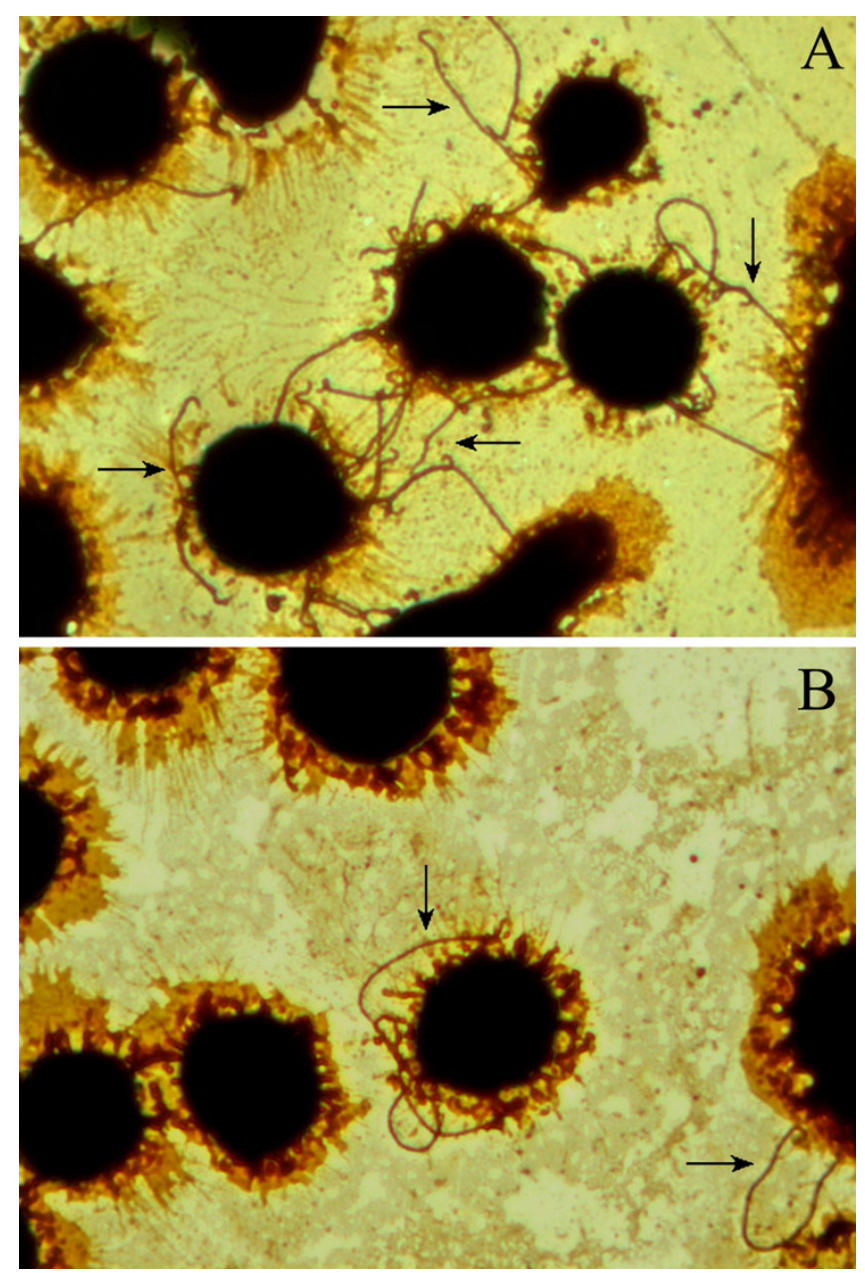

Figure 5

Adhesion of the fliY-mutant and wild-type strain to J774A.I cells. Adhesion of the wild-type strain $(A)$ and fliYmutant $(B)$. The arrow indicates the adhering leptospires on J774A.I cells. This experiment was repeated three times. Magnification $\times 400$.

genes) may decrease the export of some unknown adhesion- and cytotoxicity-associated virulence proteins.

\section{Conclusion}

Inactivation of fliY clearly had polar effects on downstream genes. The phentotypes observed, including decreasing motility, adhesion to macrophages and hostcell apoptosis, and attenuating lethality in infected guinea pigs, could be a consequence of fliY inactivation, but also a consequence of the polar effects.

\section{Methods}

\section{Bacterial strains and cell lines}

L. interrogans serogroup Icterohaemorrhagiae serovar Lai strain Lai was offered by the National Institute for the Control of Pharmaceutical and Biological Products in Beijing, China. The leptospires were cultured in Korthof liq-

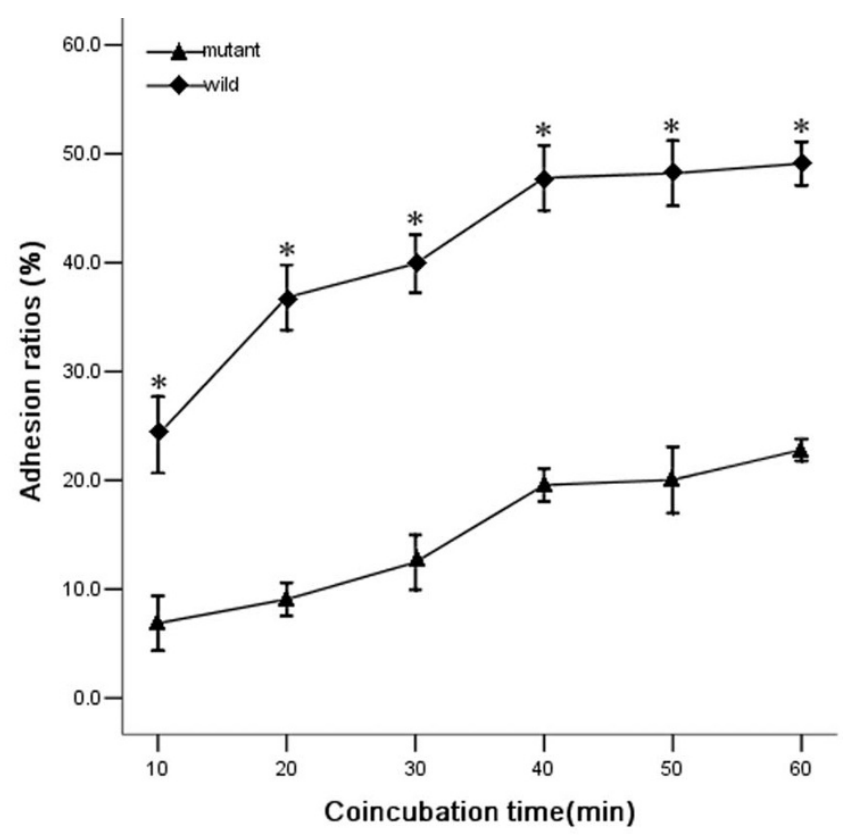

Figure 6

Adhesion ratios of the fliY-mutant and wild-type strain to J774A. I cells after different incubation

times. Adhesion was quantified as described in Methods. *: $P$ $<0.05$, wild-type strain compared with the mutant.

uid medium containing $8 \%$ heat-inactivated rabbit serum (RS) at $28^{\circ} \mathrm{C}$. To maintain virulence, the strain was passaged intraperitoneally in specific pathogen-free DunkinHartley ICO:DH (Poc) guinea pigs (2 weeks old, each weighing about $120 \mathrm{~g}$ ) before use, according to the description by Merien et al. and Viriyakosol et al. [44,54]. Animal protocols were approved by the Animal Ethics Review Committee of Zhejiang University.

\section{Cell line and culture}

The murine mononuclear-macrophage-like cell line (J774A.1) was obtained from the American Type Culture Collection (Rockville, MD, USA). The cells were cultured in RPMI 1640 medium (GIBCO, USA), supplemented with $10 \%$ heat-inactivated fetal calf serum (FCS) (GIBCO), $100 \mathrm{U} / \mathrm{ml}$ penicillin and $100 \mu \mathrm{g} / \mathrm{ml}$ streptomycin (Sigma, USA) at $37^{\circ} \mathrm{C}$ in an atmosphere of $5 \% \mathrm{CO}_{2}$.

\section{PCR and sequencing}

Genomic DNA of $L$. interrogans strain Lai was extracted using Bacterial Genomic DNA Extraction Kit (BioColor, China). Plasmid pUC19, which has an ampicillin resistant gene (bla) cassette including promotor in E. coli DH5a, was prepared by Mini-plasmid Rapid Isolation Kit (BioDev, China). Primers for amplifications of the fliY and bla genes are shown in Table 2. A commercial PCR Kit (TaKaRa, China) was used to amplify the fliY and bla genes. The products were detected on $1.5 \%$ ethidium bro- 
A Control

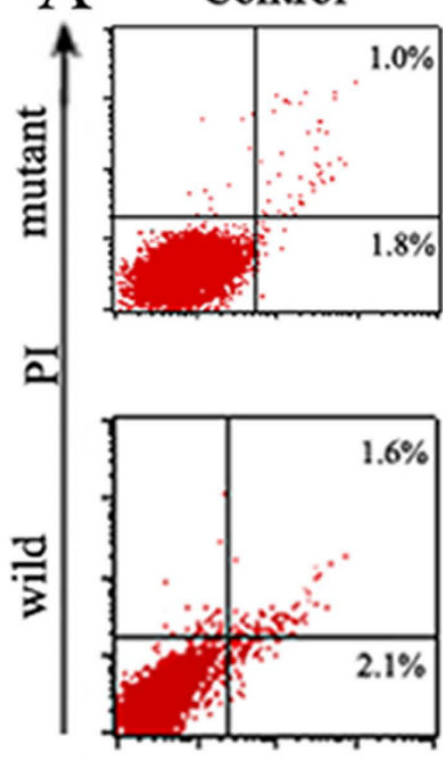

$1 \mathrm{~h}$
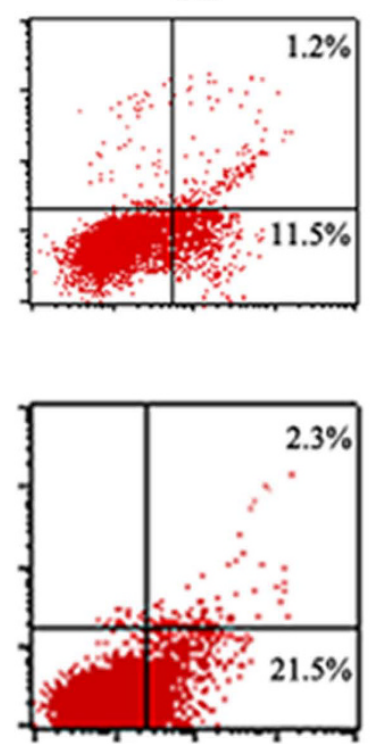

$2 \mathrm{~h}$
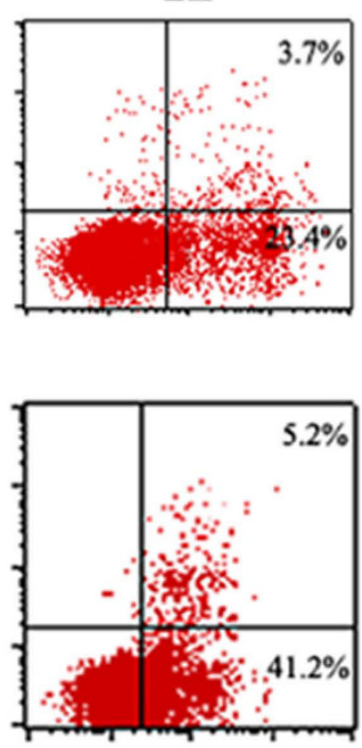

$4 \mathrm{~h}$
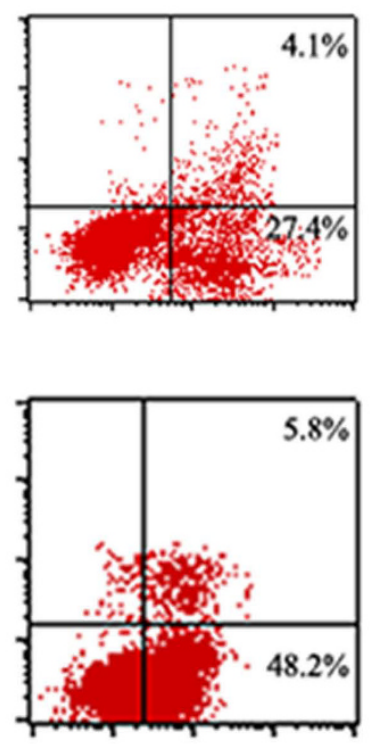

\section{Annexin V}
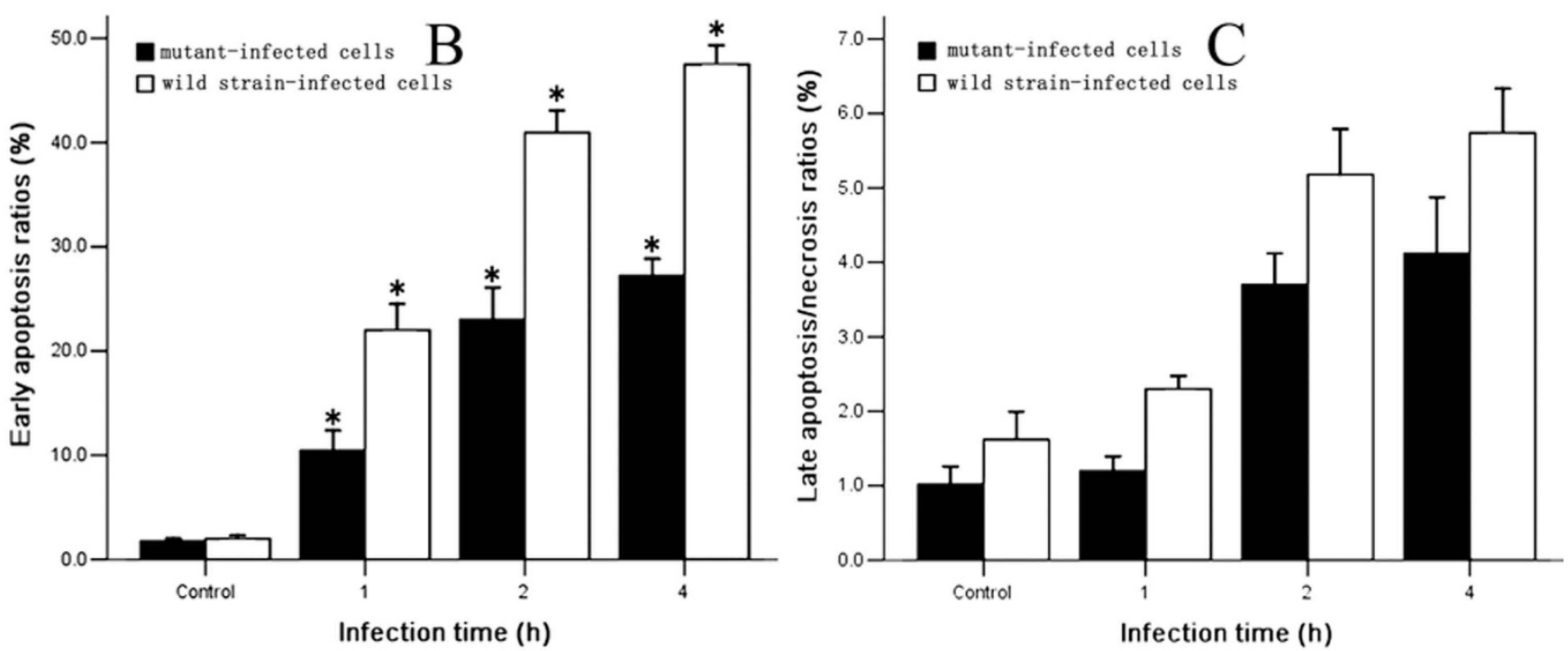

Figure 7

Apoptosis ratios of J774A.I cells induced by the fliY- mutant and wild-type strain. Panel A: lower left quadrants indicate unstained normal cells; lower right quadrants, the early apoptotic cells binding Annexin- $V$; upper left quadrants, the necrotic cells binding PI; and upper right quadrants, the late apoptotic/necrotic cells binding both Annexin- $\mathrm{V}$ and $\mathrm{PI}$. Cell death was measured by flow cytometry as described in Methods. Panel B: proportion of early apoptotic cells (annexin- $\left.\mathrm{V}^{+} / \mathrm{PI}^{-}\right)$after infection for different times. The data are expressed as mean \pm SD for three independent experiments. Panel C: proportion of late apoptotic/necrotic cells (annexin $-\mathrm{V}^{+} / \mathrm{PI}^{+}$) after infection for different times. The data are expressed as mean \pm SD for three independent experiments. $*_{:} P<0.05$, wild-type strain compared with the mutant. 
Table I: Lethality of the fliY- mutant and the wild-type strain in infected guinea pigs.

\begin{tabular}{lcccc}
\hline Strain & $\begin{array}{c}\text { Challenge dosage } \\
\left(\times 10^{8} \text { per animal) }\right.\end{array}$ & $\begin{array}{c}\text { Animal } \\
(\mathbf{n})\end{array}$ & $\begin{array}{c}\text { Dead/surviving } \\
(\mathbf{n} / \mathbf{n})\end{array}$ & $\begin{array}{c}\text { Death rate } \\
(\%)\end{array}$ \\
\hline Wild-type Mutant & 6 & 10 & $10 / 0$ & 100 \\
& 6 & 10 & $0 / 10$ & 0 \\
& 12 & 10 & $0 / 10$ & 0 \\
& 30 & 10 & $0 / 10$ & 6 \\
& 60 & 10 & $6 / 4$ & 60 \\
\hline
\end{tabular}

mide pre-stained agarose gel by electrophoresis, purified using PCR Product Purification Kit (BioColor), and ligated into plasmid pUCm-T using T-A Cloning Kit (BioColor) to form recombinant plasmids pUCm-T ${ }^{f i Y}$. pUCm$\mathrm{T}^{\text {bla }}$ sequencing was performed by Invitrogen Co. Ltd in China.

\section{Expression, purification and immunization of recombinant \\ FliY}

pUCm-T fliY $^{\prime}$ and expression vector pET32a (Novagen, USA) were digested with BamH I and Hind III, respectively. The recovered fliY segment was ligated into linearized pET32a using T4 DNA ligase (TaKaRa), and then transformed into E. coli BL21DE3 (Novagen) to form E. coli BL21DE3pET32a-fliY. Recombinant FliY (rFliY) was expressed under inducement of $0.5 \mathrm{mM}$ IPTG for $4 \mathrm{~h}$ at $37^{\circ} \mathrm{C}$. The expressed rFliY was extracted by Ni-NTA affinity chromatography and the purity of rFliY was determined by SDS-PAGE. New Zealand rabbits, provided by the Laboratory Animal Center of Zhejiang University, were immunized intradermally four times at an interval of once a week with the purified rFliY that was pre-mixed with Freund's adjuvant. On the 15th day after the last immunization, the rabbit serum was collected and the immunodiffusion test was used to examine the titer of antiserum.

\section{Generation and characterization of the fliY-mutant}

Plasmid p2NIL used in this study was kindly offered by Dr. Tanya Parish and Dr. Amanda C. Brown. The fliY segment from pUCm-T $\mathrm{T}^{f l i Y}$ was inserted into p2NIL at the
BamH I/Hind III sites to form p2NIL fliY. The plasmid has an origin of replication for E. coli (oriE), a kanamycin resistance gene (kan), and a multiple cloning site [55]. Since there is a unique $B g l$ II site within the fliY gene

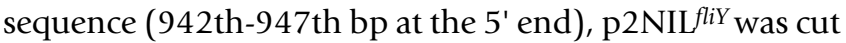
with $B g l$ II, dephosphorylated and ligated with ampicillin amplification segment (bla) including the promotor (10th-16th bp at 5 ' end) flanked by a $B g l$ II site to form a suicide plasmid, p2NIL fliY-bla. The suicide plasmid was transformed into E. coli DH5a for amplification in LuriaBertani (LB) medium supplemented with both $100 \mu \mathrm{g} / \mathrm{ml}$ ampicillin and $50 \mu \mathrm{g} / \mathrm{ml}$ kanamycin, and then recovered for sequencing. The p2NIL fliY-bla plasmid was then denatured by alkali treatment as previously described [56,57], and electrocompetent leptospires were prepared according to Saint Girons' protocol [58]. The competent leptospiral cells were mixed with $2 \mu \mathrm{g}$ p $2 \mathrm{NIL}^{\text {fliY-amp }} \mathrm{DNA}$, and then bathed on ice for $10 \mathrm{~min}$ for electrotransformation. Finally, the mixture was transferred to $1 \mathrm{ml}$ of $8 \% \mathrm{RS}$ Korthof liquid medium for a $48 \mathrm{~h}$ incubation at $28^{\circ} \mathrm{C}$. The fli $Y^{-}$mutant was selected on $8 \%$ RS Korthof plates containing $100 \mu \mathrm{g} / \mathrm{ml}$ ampicillin. Individual ampicillin-resistant colonies were inoculated in $8 \%$ RS Korthof liquid medium supplemented with $100 \mu \mathrm{g} / \mathrm{ml}$ ampicillin. The steps to construct the suicide plasmid and to generate $f l i Y^{-}$ mutant are summarized in Fig 8.

\section{Confirmation of the fliY gene inactivation in mutants}

The fli $Y^{-}$mutant was cultured at $28^{\circ} \mathrm{C}$ in $8 \%$ RS Korthof liquid medium containing $100 \mu \mathrm{g} / \mathrm{ml}$ ampicillin. Genomic DNA of the mutant was extracted using Bacterial

Table 2: Primer information for amplification of the fliY and bla genes.

\begin{tabular}{llc}
\hline Gene & Primer sequence (5'-3') & Product size \\
\hline fliY & F: GCC GGA TCC $($ BamH I) ATG GGT GAA GGT TCC CTA TCA CAG & 1065 bP \\
& R: GCC AAG CTT $($ Hind III) TCA CTT ACC CTC CGG CTT AAT CCG & 954 bP \\
bla & F: GCC AGA TCT $($ Bgl II) TCT AAA TAC ATT CAA ATA TGT & \\
& R: GCC AGA TCT $($ Bgl II) CTT GGT CTG ACA GTT ACC AAT & 804 bP \\
flip & F: ATG AAA ATG AGA CAT AAA & 264 bP \\
& R: TCA TTT ATA ACT CCT TAC & F: ATG ACG GAA TTA GAC GTT ATG \\
& R: CTA AAA TTT TTC GAT CAT CAA & \\
\hline
\end{tabular}




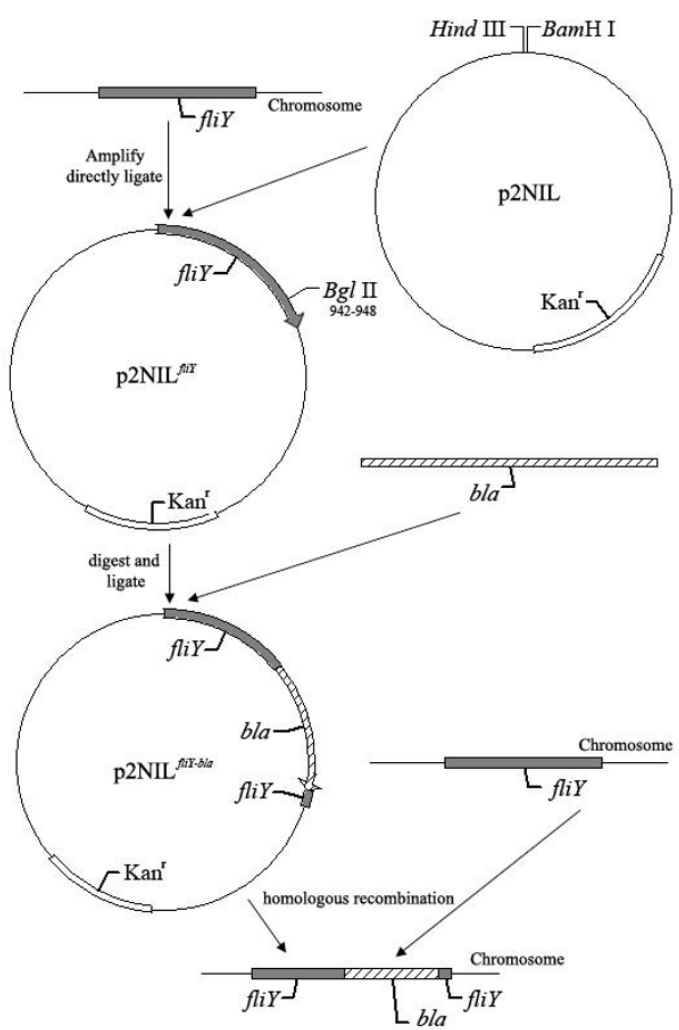

Figure 8

Strategy for preparing the fliY-mutant using the suicide plasmid p2NIL fliy-bla.

Genomic DNA Extraction Kit (BioColor), and the disrupted fliY gene in the mutant was identified by PCR and the Western Blot assay. The product of the fliY-bla gene is larger in the mutant $(2019 \mathrm{bp})$ than the fliY gene in the wild-type strain (1065 bp). By using 1:2500 diluted antirFliY serum as the primary antibody and 1:3000 diluted HRP-labeling goat anti-rabbit IgG (Jackson ImmunoResearch Laboratories, USA) as the secondary antibody, a Western Blot assay was performed to detect the expression of FliY protein in the mutant. In the genomic sequence of L. interrogans serovar Lai strain Lai, the fliP and fliQ genes are located downstream from the fliY gene. In order to further confirm the inactivation of the fliY gene, two separate RT-PCRs were performed to detect mRNAs of the fliP and fliQ genes, with primers shown in table 2. In addition, an operon predictor tool http://www.microbesonline.org/ was used for analysis of the operon structure.

\section{Motility assay}

The motility and shapes of the fliY- mutant and wild-type strain in $8 \%$ RS Korthof liquid medium were observed under dark-field microscope after incubation at $28^{\circ} \mathrm{C}$ for $10 \mathrm{~d}$ (the primary generation), $50 \mathrm{~d}$ (the 5 th generation) and $100 \mathrm{~d}$ (the 10th generation). The colony sizes of the mutant and wild-type strain on $8 \%$ RS semisolid Korthof plate $(0.25 \%$ agar $)$ that had been incubated at $28^{\circ} \mathrm{C}$ for three weeks were measured for three times as described above.

\section{Fontana silver staining}

J774A. 1 cells $\left(5 \times 10^{4}\right.$ cells $\left./ \mathrm{ml}\right)$ were seeded on coverslips in 12-well tissue culture plates (Corning, USA) and preincubated for $24 \mathrm{~h}$ at $37^{\circ} \mathrm{C}$ in an atmosphere of $5 \% \mathrm{CO}_{2}$. The freshly cultured leptospires of the fliY- mutant and wild-type strain were harvested by centrifugation $(12,000$ $\times \mathrm{g}, 15 \mathrm{~min}, 15^{\circ} \mathrm{C}$ ) and washed twice with autoclaved PBS. The pellets were suspended in pre-warmed antibiotics-free $10 \%$ FCS RPM1640 to a final concentration of $10^{8}$ leptospires/ml by dark-field microscopy with a Petroff-Hausser counting chamber (Fisher Scientifics, PA). The cell monolayers were washed three times with autoclaved PBS and then infected with each of the suspensions at an MOI of 100 (100 leptospires per cell) for 10, 20, 30, 40, 50 and 60 min, respectively. After infection, the coverslips were washed three times with PBS to remove non-adherent leptospires, fixed in 5\% formaldehyde, stained with silver nitrate, and observed under a light microscope [59]. The adhesion ratio was defined as the number of adhering leptospires per 100 infected host-cells $\times 100 \%$ [11].

\section{Assessment of cell death by flow cytometry}

Apoptosis was measured by flow cytometry using annexin-V-fluorescein isothiocyanate (FITC)/propidium iodide (PI) labeling as previously published $[11,60]$. The J774A.1 cell monolayers were infected with either the fli $\mathrm{Y}^{-}$ mutant or wild-type strain with an MOI of 100 at $37^{\circ} \mathrm{C}$ for 1,2 , or $4 \mathrm{~h}$ [46]. After infection, the cells were washed three times with PBS, collected with a cell scratcher, and centrifuged at $1,000 \times \mathrm{g}$ for $5 \mathrm{~min}$. The pellets were washed three times with PBS, resuspended in annexin-V binding buffer with FITC-conjugated annexin-V, and incubated for $15 \mathrm{~min}$ at room temperature in the dark, following the manufacturer's instructions (Caltag Laboratories, USA). After PI was added, the cell suspension was immediately analyzed by FACSCalibur flow cytometry and CellQuest Pro software (Beckman Coulter, USA). Cells in the early apoptotic phase bind annexin- $\mathrm{V}$ but exclude PI, and those in the late apoptotic/necrotic phase stain with both annexin-V and PI, while necrotic cells stain with PI alone [60].

\section{Animals and challenge infections}

The Dunkin-Hartley guinea pigs ( $150 \pm 5 \mathrm{~g}$, 3 weeks old) used in this study were provided by the Laboratory Animal Center of Zhejiang University. The animals were challenged intraperitoneally with different dosages of either wild-type L. interrogans serovar Lai strain Lai or the fliY- 
mutant, and then observed for $10 \mathrm{~d}$ [1]. The animal experiments were approved by the Animal Ethics Review Committee of Zhejiang University.

\section{Statistical analysis}

Data from a minimum of three experiments were averaged and presented as mean \pm SD (standard deviation). One-way analysis of variance (ANOVA) followed by the Dunnett's multiple comparisons test were used to determine significant differences. Statistical significance was defined as $P$ value $\leq 0.05$.

\section{Authors' contributions}

SL carried out the molecular genetic studies, immunoassays and drafted the manuscript. AS cultured the leptospires and participated in immunoassays. DMO participated in study design and revised the manuscript. SW and JZ carried out analysis and interpretation of data. JY conceived of the study, and participated in its design and coordination. All authors read and approved the final manuscript, and agreed to having it published.

\section{Acknowledgements}

This work was supported by a Grant (30370072) from the National Natural Science Foundation of China and a grant (2007XZA02) from the Natural Scientific National Foundation of Zhejiang Medical College of China. We are grateful to Dr. Tanya Parish and Dr. Amanda C. Brown (Center for Infectious Disease, Institute for Cell and Molecular Science, Queen Mary's School of Medicine and Dentistry, UK) for having graciously provided the plasmid p2NIL used in this study.

\section{References}

I. Faine S, Adher B, Bloin C, Perolat P: Leptospira and leptospirosis. 2nd edition. Australia: MedSci; 1999.

2. Bharti AR, Nally JE, Ricaldi JN, Matthias MA, Diaz MM, Lovett MA, Levett PN, Gilman RH, Willig MR, Gotuzzo E, Vinetz JM: Leptospirosis: a zoonotic disease of global importance. Lancet Infect Dis 2003, 3:757-77I.

3. McBride AJ, Athanazio DA, Reis MG, Ko Al: Leptospirosis. Curr Opin Infect Dis 2005, I 8:376-386.

4. Lomar AV, Diament D, Torres JR: Leptospirosis in Latin America. Infect Dis Clin N Am 2000, 14:23-39. vii-viii

5. Levett PN: Leptospirosis. Clin Microbio Rev 200I, 14:296-326.

6. Meslin FX: Global aspects of emerging and potential zoonoses: a WHO perspective. Emerg Infect Dis 1997, 3:223-228.

7. Brooks GF, Butel JS, Morse SA: Medical Microbiology. 22nd edition. U.S.A.: McGraw-Hill; 200I:29I-293.

8. Wolgemuth CW, Charon NW, Goldstein SF, Goldstein RE: The flagellar cytoskeleton of the spirochetes. J Mol Microbiol Biotechnol 2006, II:22I-227.

9. Li C, Motaleb A, Sal M, Goldstein SF, Charon NW: Spirochete periplasmic flagella and motility. Mol Microbiol Biotechnol 2000, 2:345-354.

10. Charon NW, Goldstein SF: Genetics of motility and chemotaxis of a fascinating group of bacteria: the spirochetes. Annu Rev Genet 2002, 36:47-73.

11. Li LW, Ojcius DM, Yan J: Comparison of invasion of fibroblasts and macrophages by high- and low-virulence Leptospira strains: colonization of the host-cell nucleus and induction of necrosis by the virulent strain. Arch Microbiol 2007, 188:59I-598.

12. Dong HY, Hu Y, Xue F, Sun D, Ojcius DM, Mao YF, Yan J: Characterization of the ompLI gene of pathogenic Leptospira species in China and cross-immunogenicity of the OmpLI protein. BMC Microbiol 2008, 8:223-235.
13. Macnab RM: How bacteria assemble flagella. Ann Rev Microbiol 2003, 57:77-100.

14. McCarter LL: Regulation of flagella. Curr Opin Microbiol 2006, 9:180-186.

15. Francis NR, Irikura VM, Yamaguchi S, DeRosier DJ, Macnab RM: Localization of the Salmonella typhimurium flagellar switch protein FliG to the cytoplasmic M-ring face of the basal body. Proc Natl Acad Sci USA 1992, 89:6304-6308.

16. Zhao RPN, Jaffe H, Reese TS, Khan S: FliN is a major structural protein of the C-ring in the Salmonella typhimurium flagellar basal body. Mol Biol 1996, 261:195-208.

17. Thomas DR, Morgan DG, DeRosier DJ: Rotational symmetry of the $\mathrm{C}$ ring and a mechanism for the flagellar rotary motor. Proc Natl Acad Sci USA 1999, 96:10134-10139.

18. Kojima S, Blair DF: The bacterial flagellar motor: structure and function of a complex molecular machine. Inter Rev Cytol 2004, 233:93-134.

19. Ren SX, Fu G, Jiang XG, Zeng R, Miao YG, Xu H, Zhang YX, Xiong $H$, Lu G, Lu LF, Jiang HQ, Jia J, Tu YF, Jiang JX, Gu WY, Zhang YQ, Cai Z, Sheng HH, Yin HF, Zhang Y, Zhu GF, Wan M, Huang HL, Qian Z, Wang SY, Ma W, Yao ZJ, Shen Y, Qiang BQ, Xia QC, Guo XK, Danchin A, Saint Girons I, Somerville RL, Wen YM, Shi MH, Chen Z, Xu JG, Zhao GP: Unique physiological and pathogenic features of Leptospira interrogans revealed by whole-genome sequencing. Nature 2003, 422:888-893.

20. Nascimento AL, Ko Al, Martins EA, Monteiro-Vitorello CB, Ho PL, Haake DA, Verjovski-Almeida S, Hartskeerl RA, Marques MV, Oliveira MC, Menck CF, Leite LC, Carrer H, Coutinho LL, Degrave WM, Dellagostin OA, El-Dorry H, Ferro ES, Ferro MI, Furlan LR, Gamberini M, Giglioti EA, Góes-Neto A, Goldman GH, Goldman MH, Harakava R, Jerônimo SM, Junqueira-de-Azevedo IL, Kimura ET, Kuramae EE, Lemos EG, Lemos MV, Marino CL, Nunes LR, de Oliveira RC, Pereira GG, Reis MS, Schriefer A, Siqueira WJ, Sommer P, Tsai SM, Simpson AJ, Ferro JA, Camargo LE, Kitajima JP, Setubal JC, van Sluys MA: Comparative genomics of two Leptospira interrogans serovars reveals novel insights into physiology and pathogenesis. J Bacteriol 2004, 186:2164-2172.

21 . Szurmant $\mathrm{H}$, Ordal GW: Diversity in chemotaxis mechanisms among the bacteria and archaea. Microbiol Mol Biol Rev 2004, 68:301-319.

22. Szurmant H, Muff TJ, Ordal GW: Bacillus subtilis CheC and FliY are members of a novel class of CheY-P-hydrolyzing proteins in the chemotactic signal transduction cascade. J Biol Chem 2004, 279:21787-21792.

23. Straley SC, Skrzypek E, Plano GV, Bliska JB: Yops of Yersinia spp. pathogenic for humans. Infect Immun 1993, 61:3105-3110.

24. Fields KA, Plano GV, Straley SC: A low-Ca2+ response (LCR) secretion (ysc) locus lies within the IcrB region of the LCR plasmid in Yersinia pestis. J Bacteriol 1994, I76:569-579.

25. Tang H, Billings S, Wang X, Sharp L, Blair DF: Regulated underexpression and overexpression of the FliN protein of Escherichia coli and evidence for an interaction between FliN and FliM in the flagellar motor. J Bacteriol 1995, I77:3496-3503.

26. Arora SK, Ritchings BW, Almira EC, Lory S, Ramphal R: The Pseudomonas aeruginosa flagellar cap protein, FliD, is responsible for mucin adhesion. Infect Immun 1998, 66: 1000-1007.

27. Ottemann KM, Lowenthal AC: Helicobacter pylori uses motility for initial colonization and to attain robust infection. Infect Immun 2002, 70: 1984-1990.

28. Mahajan A, Currie CG, Mackie S, Tree J, McAteer S, McKendrick I, McNeilly TN, Roe A, La Ragione RM, Woodward MJ, Gally DL, Smith DG: An investigation of the expression and adhesin function of $\mathrm{H7}$ flagella in the interaction of Escherichia coli O 157:H7 with bovine intestinal epithelium. Cell Microbiol 2009, II:121-137.

29. Weinstein DL, Carsiotis M, Lissner CR, O'Brien AD: Flagella help Salmonella typhimurium survive within murine macrophages. Infect Immun 1984, 46:819-825.

30. Liu SL, Ezaki T, Miura H, Matsui K, Yabuuchi E: Intact motility as a Salmonella typhi invasion-related factor. Infect Immun 1988, 56: 1967-1973.

31. Dai B: Advances in research on leptospira and human leptospirosis in China. Chin Med Sci J 1992, 7:239-243.

32. Croda J, Figueira CP, Wunder EA Jr, Santos CS, Reis MG, Ko Al, Picardeau M: Targeted mutagenesis in pathogenic Leptospira 
species: disruption of the LigB gene does not affect virulence in animal models of leptospirosis. Infect Immun 2008, 76:5826-5833

33. Bischoff DS, Ordal GW: Identification and characterization of FliY, a novel component of the Bacillus subtilis flagellar switch complex. Mol Microbiol 1992, 6:27I 5-2723.

34. Senesi S, Celandroni F, Salvetti S, Beecher DJ, Wong AC, Ghelardi E: Swarming motility in Bacillus cereus and characterization of a fliY mutant impaired in swarm cell differentiation. Microbiology 2002, I 48: I 785- 1794

35. Nougayre JP, Fernandes PJ, Donnenberg MS: Adhesion of enteropathogenic Escherichia coli to host cells. Cell Microbiol 2003, 5:359-372.

36. Kline KA, Fälker S, Dahlberg S, Normark S, Henriques-Normark B: Bacterial adhesins in host-microbe interactions. Cell Host Microbe 2009, 5:580-592.

37. Cinco M, Domenis R, Perticarari S, Presani G, Marangoni A, Blasi E. Interaction of leptospires with murine microglial cells. New Microbiol 2006, 29:193-199.

38. Choy HA, Kelley MM, Chen TL, Moller AK, Matsunaga J, Haake DA Physiological osmotic induction of Leptospira interrogans adhesion: LigA and LigB bind extracellular matrix proteins and fibrinogen. Infect Immun 2007, 75:244 I-2450.

39. Coburn J, Fischer JR, Leong JM: Solving a sticky problem: new genetic approaches to host cell adhesion by the Lyme disease spirochete. Mol Microbiol 2005, 57: I | 82-I I 95.

40. Edwards AM, Jenkinson HF, Woodward MJ, Dymock D: Binding properties and adhesion-mediating regions of the majo sheath protein of Treponema denticola ATCC 35405 . Infect Immun 2005, 73:289I-2898.

41. Monack DM, Raupach B, Hromockyj AE, Falkow S: Salmonella typhimurium invasion induces apoptosis in infected macrophages. Proc Natl Acad Sci USA 1996, 93:9833-9838.

42. Mills SD, Boland A, Sory MP, Smissen P van der, Kerbourch C, Finlay BB, Cornelis GR: Yersinia enterocolitica induces apoptosis in macrophages by a process requiring functional type III secretion and translocation mechanisms and involving YopP, presumably acting as an effector protein. Proc Natl Acad Sci USA 1997, 94: | 2638-| 2643.

43. Albee L, Shi B, Perlman H: Aspartic protease and caspase 3/7 activation are central for macrophage apoptosis following infection with Escherichia coli. J Leukoc Biol 2007, 81:229-237.

44. Merien F, Baranton G, Perolat P: Invasion of Vero cells and induction of apoptosis in macrophages by pathogenic Leptospira interrogans are correlated with virulence. Infect Immun 1997 65:729-738.

45. Liu YY, Zheng W, Li LW, Mao Y, Yan J: Pathogenesis of leptospirosis: interaction of Leptospira interrogans with in vitro cultured mammalian cells. Med Microbiolo Immunol 2007, 1 96:233-239.

46. Jin DD, Ojcius DM, Sun D, Dong HY, Luo YH, Mao YF, Yan J: Leptospira interrogans induces apoptosis in macrophages via caspase-8- and caspase-3-dependent pathways. Infect Immun 2009, 77:799-809.

47. Hueck C): Type III protein secretion systems in bacterial pathogens of animals and plants. Microbiol Mol Bio Rev 1998, 62:379-433

48. Stuber K, Frey J, Burnens AP, Kuhnert P: Detection of type III secretion genes as a general indicator of bacterial virulence. Mole Cell Probes 2003, I 7:25-32.

49. Kubori T, Matsushima Y, Nakamura D, Uralil J, Lara-Tejero M, Sukhan A, Galán JE, Aizawa SI: Supramolecular structure of the Salmonella typhimurium type III protein secretion system. Science 1998, 280:602-605.

50. Young GM, Schmiel DH, Miller VL: A new pathway for the secretion of virulence factors by bacteria: the flagellar export apparatus functions as a protein-secretion system. Proc Natl Acad Sci USA 1999, 96:6456-646I.

51. Arora SK, Ritchings BW, Almira EC, Lory S, Ramphal R: Cloning and characterization of Pseudomonas aeruginosa flif, necessary for flagellar assembly and bacterial adherence to mucin. Infect Immun 1996, 64:2 I 30-2136.

52. Mecsas J], Strauss EJ: Molecular mechanisms of bacterial virulence: type III secretion and pathogenicity islands. Emerg Infect Dis 1996, 2(4):270-288.
53. Warren SM, Young GM: An amino-terminal secretion signal is required for YpIA export by the Ysa, Ysc, and flagellar type III secretion systems of Yersinia enterocolitica biovar IB. J Bacteriol 2005, I 87: | 430-40.

54. Viriyakosol S, Matthias MA, Swancutt MA, Kirkland TN, Vinetz JM: Toll-like receptor 4 protects against lethal Leptospira interrogans serovar Icterohaemorrhagiae infection and contributes to in vivo control of leptospiral burden. Infec Immun 2006, 74:887-895.

55. Parish T, Stoker NG: Use of a flexible cassette method to generate a double unmarked Mycobacterium tuberculosis tyA plcABC mutant by gene replacement. Microbiology 2000, I46:1969-1975.

56. Hinds J, Mahenthiralingam E, Kempsell KE, Duncan K, Stokes RW, Parish T, Stoker NG: Enhanced gene replacement in mycobacteria. Microbiology 1999, I45:519-527.

57. Picardeau M, Brenot A, Saint Girons I: First evidence for gene replacement in Leptospira spp. inactivation of $L$. biflexa flaB results in non-motile mutants deficient in endoflagella. Mol Microbiol 2001, 40:189-199.

58. Saint Girons I, Bourhy P, Ottone C, Picardeau M, Yelton D, Hendrix RW, Glaser P, Charon N: The LEI bacteriophage replicates as a plasmid within Leptospira biflexa: construction of an L. biflexa-Escherichia coli shuttle vector. J Bacteriol 2000 , I 82:5700-5705.

59. Saravanan R, Rajendran P, Thyagarajan SP, Smythe LD, Norris MA Symonds ML, Dohnt MF: Leptospira autumnalis isolated from a human case from Avadi, India, and the serovar's predominance in local rat and bandicoot populations. Ann Trop Med Parasitol 2000, 94:503-506.

60. Perfettini JL, Gissot M, Souque P, Ojcius DM: Modulation of apoptosis during infection with Chlamydia. Methods Enzymol 2002 358:334-344.
Publish with Bio Med Central and every scientist can read your work free of charge

"BioMed Central will be the most significant development for disseminating the results of biomedical research in our lifetime. "

Sir Paul Nurse, Cancer Research UK

Your research papers will be:

- available free of charge to the entire biomedical community

- peer reviewed and published immediately upon acceptance

- cited in PubMed and archived on PubMed Central

- yours - you keep the copyright
BiolMedcentral 